Wir dokumentieren im Folgenden den leicht gekürzten Vorschlag für eine alternative Gesundheitspolitik der Internetzeitschrift www.links-netz.de. Bereits 2003 legten die Verfasser_innen in einem Grundlagentext ein Gegenprojekt zur neoliberalen Sozialpolitik vor. Dieser Text wurde in diesem Jahr, nach sieben Jahren Debatte, ${ }^{1}$ aktualisiert. ${ }^{2}$ Gerade weil die Krisenhaftigkeit des Neoliberalismus die traditionelle, fordistische soziale Sicherung immer weiter aushebele, sei es umso dringlicher, Sozialpolitik an einer anderen Logik zu orientieren. „Sozialpolitik als soziale Infrastruktur" stelle diese andere Logik dar. Sie sieht die Aufgabe von Sozialpolitik darin, die Voraussetzungen für das gute Leben aller zu schaffen. Ausgangspunkt ist dabei eine Erweiterung des Arbeitsbegriffes, der nicht mehr nur auf Erwerbsarbeit reduziert werden, sondern darüber hinaus alle Formen, besonders Hausarbeit, Eigenarbeit und freiwillige Arbeit umfassen soll. Die soziale Infrastruktur müsse in diesem Kontext allen zur Verfügung stehen, völlig unabhängig von vorherigen Einzahlungen oder nachfolgenden Verpflichtungen. „Infrastruktur ist alles, was die Haushalte an Leistungen brauchen, damit alle Beteiligten gut leben und sich entwickeln können, das sie aber nicht selbst produzieren können. “3 Es gibt inzwischen zwei Ausarbeitungen des Grundlagenpapiers in konkreten Sozialpolitikbereichen (Gesundheit, Bildung); angesichts der aktuellen Debatte um die sogenannte Kopfpauschale dokumentieren wir das Konzept für die Gesundheitspolitik. Die Redaktion.

\title{
Sozialpolitik als Infrastruktur - der Gesundheitsbereich
}

\section{AG links-netz, Textfassung: Joachim Hirsch, Eva-Maria Krampe, Christa Sonnenfeld und Heinz Steinert*}

„(...) Die Konzepte zur Sozialpolitik, die wir in die Diskussion gebracht haben, sollen Anstöße zu einem radikalen Umdenken geben. Sie sind Lockerungsübungen in einer festgefahrenen (wie immer auch „linken“) Politik, der die Ideen ausgegangen sind. Wir wollen nützliche Fragen aufwerfen, unter anderem solche, die mit „warum eigentlich nicht...? “ beginnen.

Das Konzept einer Sozialpolitik als Bereitstellen der „Infrastruktur des guten Lebens aller" ist einerseits unrealistisch genug, dass die Wirklichkeit sich an ihm blamieren kann, andererseits plausibel genug, um die Frage aufzuwerfen, was die real existierende Sozialpolitik eigentlich stattdessen anstrebt. Die Diskussion des allgemeinen Modells, die wir mit anregen konnten und die immer weitere Kreise zieht, ist interessant, hat sich aber - für unsere Intention zu einseitig - auf verschiedene Formen eines garantierten Grundeinkommens eingeengt. Wir haben von Beginn an darauf hingewiesen, dass das Grundeinkommen im Zusammenhang einer sehr viel weiter greifenden Einrichtung von sozialer Infrastruktur ge-

1 http://www.links-netz.de/rubriken/R_infrastruktur.html.

2 http://www.links-netz.de/K_texte/K_links-netz_sozpol.html.

3 http://www.links-netz.de/rubriken/R_infrastruktur.html.

Nadja Rakowitz wird für ihre fachkundige Unterstützung gedankt. 
sehen werden muss. Dieser Begriff bezieht sich auf ein gesellschaftliches Arrangement, das für alle Anwesenden die zu einem menschenwürdigen Leben notwendigen Güter und Dienste bereithält. Das sind Einrichtungen der Gesundheitssicherung, der Bildung, des Transports, schließlich des Wohnens und Essens, die allgemein zur Verfügung stehen und im Bedarfsfall benutzt werden können. Zuwendungen an Einzelne sind nur in zweiter Linie notwendig, und das vor allem deshalb, weil Wahlfreiheit und Autonomie der Lebensführung ermöglicht werden sollen. Geld ist das universelle Mittel dafür. Daher ist die Infrastruktur zu ergänzen und zugänglich zu machen durch ein bedingungsloses Grundeinkommen für alle - das umso niedriger sein kann, je besser die Infrastruktur funktioniert. Das Grundeinkommen ersetzt alle bisherigen Geldleistungen aus der Sozialversicherung und Fürsorge und ist daher in erster Linie eine enorme Verwaltungs-Vereinfachung und -Einsparung. ${ }^{4}$

Um unsere Vorstellung einer sozialen Infrastruktur zu verdeutlichen, haben wir diese für verschiedene Bereiche deutlicher ausgeführt als das im Grundsatzpapier und in der Diskussion dazu möglich war. Hier wollen wir einen Anstoß dazu geben, Vorstellungen davon zu entwickeln und zu konkretisieren, wie ein demokratisches, soziales und menschenwürdiges Gesundheitssystem aussehen könnte. Wir stellen dazu auch ein Organisationsmodell, das „lokale Gesundheitszentrum", zur Diskussion, dessen Details in einer breiteren Diskussion weiter auszuarbeiten wären.

Wie in allen Bereichen der sozialen Sicherung wird auch in der Gesundheitssicherung das bestehende System durch den Übergang zur Produktionsweise eines globalen neoliberalen Kapitalismus ausgehebelt. Gesundheitssystem und Gesundheitsrisiko bzw. Krankheit werden rasant privatisiert und vertikal ausdifferenziert. Das wird laut Koalitionsvereinbarungen der CDU-FDP-Regierung nun noch rücksichtsloser vorangetrieben. Ein Zurück zu den überkommenen korporatistisch-bürokratischen Formen des Sozialstaats ist wegen der Veränderung der Arbeitsverhältnisse, aber auch wegen ihrer ausgrenzenden und disziplinierenden Wirkungen keineswegs eine Alternative. Der keynesianisch-korporatistische Sozialstaat des Fordismus, der die gesellschaftlichen Existenzmöglichkeiten im Wesentlichen an das Lohnarbeitsverhältnis gebunden hat, gehört der Vergangenheit an. Es ist also höchste Zeit, über eine völlige Neugestaltung des Sozialen nachzudenken.

\section{Zur Kritik der aktuellen Entwicklungen}

\section{(...)}

Angesichts dieser politischen Strategien wird das Nachdenken über neue Strukturen und Modelle von Initiativen und politischen Zusammenhängen außerhalb der etablierten politischen Apparate und Machtkomplexe ausgehen müssen. Dabei gilt es, Formen der sozialen Sicherung anzuvisieren, die zu einer emanzipativen Veränderung der herrschenden Vergesellschaftungsform insgesamt beitragen. Dazu gehört es, dass die bestehende Gesellschaft entsprechend ihren produktiven Möglichkeiten allen Menschen in gleicher Weise nicht nur eine materielle Existenzsicherung, sondern auch eine umfassende medizinische Versorgung unabhängig vom Einkommen und der Art der Tätigkeit, von Alter, Geschlecht und Staatsbürgerschaft garantieren kann und soll.

4 Wahrscheinlich ist dieser Abbau von Verwaltung, der damit möglich würde, das größte Hindernis für die Durchsetzung eines Grundeinkommens, größer als die Angst davor, dass dann „niemand mehr arbeiten würde“: Einen solchen Verlust von Macht (und Posten) lässt sich die Bürokratie nicht kampflos gefallen. 
Will man die Gesundheitssicherung grundlegend neu und anders organisieren, muss man sich die real existierende, aus den Macht- und Interessen-Blöcken der Kliniken, niedergelassenen ÄrztInnen, kassenärztlichen Vereinigungen und Krankenkassen historisch entstandene Organisation wegdenken. Bei solchen Überlegungen sind jedoch einige Besonderheiten zu berücksichtigen, die jedes Gesundheitssystem charakterisieren. Dazu gehört vor allem das strukturell ungleiche Verhältnis zwischen den ExpertInnen, d.h. ForscherInnen, EntwicklerInnen, ÄrztInnen, ApothekerInnen auf der einen, den PatientInnen auf der anderen Seite. Erstere definieren Krankheiten und steuern den Bedarf an Diagnoseeinrichtungen, Therapien und Medikamenten, ohne dass den Betroffenen ausreichende Informationen über Notwendigkeiten, Möglichkeiten und Kosten zur Verfügung stehen. Letztere, also die PatientInnen, sind per definitionem in irgendeiner Weise eingeschränkt, krank, haben Schmerzen und befinden sich darum in psychisch schwierigen Situationen. Auf Grund dessen sind sie von Hilfe abhängig und auf den Schutz der Gesellschaft angewiesen. Stattdessen sollen sie in krankheitsbedingt schwierigen Situationen auf dem Markt als „KundInnen“ agieren und unter Abwägung von konkurrierenden Angeboten das „beste Angebot" auswählen.

Infrastruktur im Gesundheitsbereich umfasst ein weites Feld. Es geht dabei grundsätzlich darum, die gesellschaftlichen Verhältnisse so einzurichten, dass Gesundheitsschädigungen nach Möglichkeit vermieden werden, also um umfassende Krankheitsprävention, und darüber hinaus darum, dass körperliche Handlungsfähigkeit oder gar körperliches Wohlbefinden gesichert sind. Dabei spielen Ernährung, Wasserversorgung, Hygiene, Kanalisation, Seuchenprävention, Friedenssicherung und die Vermeidung von Berufskrankheiten und Unfällen die entscheidende Rolle. Heute kommt die Vermeidung von Umweltbelastungen aller Art hinzu. ${ }^{5}$ Fortschritte der Medizin im engeren Sinn, also die Erweiterung des Spektrums der medikamentösen oder operativen Eingriffe, sind dem nur bedingt gleichrangig. Es geht dabei darum, Verhältnisse zu schaffen, die ein selbständiges, aktives und schmerzarmes Leben für alle als Voraussetzung gesellschaftlicher Teilhabe ermöglichen.

So falsch es ist, die Erhaltung von Gesundheit $\mathrm{zu}$ einem rein medizinischen Problem zu machen - sie ist vielmehr unter anderem Aufgabe der Produktionsgestaltung und Arbeitsorganisation, der Ökologie-Politik, der Stadt- und Verkehrsplanung, Friedenspolitik -, so wichtig ist es andererseits, eine Organisation der Medizin anzustreben, deren erste Aufgabe das Erhalten der Gesundheit ist. Das Heilen von Krankheiten und Verletzungen ist daher als Folge eines Versagens bei der Verfolgung dieses Ziels zu betrachten. Eine blühende Wirtschaft ist

5 Gegen die geläufige Art der „Prävention“, die mit moralischen oder sogar strafrechtlichen Sanktionen von der Verpflichtung, die eigene Gesundheit zu pflegen, über das Rauchverbot bis zum genetischen Screening - beim Einzelnen ansetzt, muss man sich freilich zur Wehr setzen. Dass „Prävention“ als Legitimation für repressive Maßnahmen verwendet wird, sollte andererseits nicht dazu führen, dass man vernünftige Maßnahmen zur Verhinderung von Schäden und unnötigen Konfliktsituationen gleich mit ablehnt. Es geht um die „Verhinderung von Situationen“ und es darf nicht um die „Abschaffung von Personen“ gehen. Außerdem ist bei Prävention zu bedenken, dass präventive Maßnahmen oft klassenoder schichtenspezifisch angenommen werden und gerade bei „unteren“ sozialen Schichten, deren Gesundheit besonders gefährdet ist und die es deshalb am nötigsten hätten, kaum ankommen (vgl. Rolf Rosenbrock). Will man außerdem an wesentlichen Ursachen von Krankheit ansetzen, dann muss auch über betriebliche Gesundheitsförderung gesprochen werden. Diese ist zwar inzwischen gesetzlich verankert, wird aber kaum umgesetzt, weil sie so gut wie gar nicht kontrolliert wird. 
im günstigen Fall ein Mittel dazu, de facto jedoch oft gerade die Ursache der

Schäden, die vermieden werden sollen.

Zugleich sollte man sich nicht der Illusion hingeben, Verletzungen, Krankheiten, mehr oder weniger lang andauernde Zustände der körperlichen und seelischen Beeinträchtigung ließen sich ganz vermeiden oder auf lange Frist überwinden. Situationen von Schwäche und Abhängigkeit wird es immer wieder geben (sie stellen Anteile des Lebenslaufs dar; man denke an Kindheit und Alter). Daher ist der möglichst gute und für alle Beteiligten schonende Umgang mit Abhängigkeit, also die Pflege, das Zentrum des Umgangs mit Gesundheit und Krankheit. Die Ebenen, mit denen man sich als Gesundheits-Infrastruktur beschäftigen muss, sind: a/ Erhalten von Gesundheit; b/ Pflege im Fall von Schwäche und Abhängigkeit; c/ Heilen von Krankheiten - und zwar in dieser Reihenfolge.

\section{Wenn aus PatientInnen so genannte KundInnen werden und Pflegebedürftige von Familienangehörigen oder für teures Geld versorgt werden}

\section{(...)}

Das von der rot-grünen Regierung 2004 eingerichtete Institut für Qualität und Wirtschaftlichkeit im Gesundheitswesen (IQWIG) ${ }^{6}$ stellt einen Versuch zu mehr Kontrolle u.a. der Pharmaindustrie dar. Die jetzige Regierung macht sich allerdings gerade daran, das Institut wieder zu entmachten, da es ihr zu unabhängig war und in seinen Gutachten und Entscheidungen eine pharmakritische Position gezeigt hat.

Eine inzwischen in einigen peripheren Ländern versuchte Möglichkeit ist die Herstellung von Generika - trotz geltender Patente. Laut dem geltenden TRIPSAbkommen der Welthandelsorganisation ist dies allerdings nur wenigen „least developed countries“ erlaubt. Diese Regelung soll 2016 auslaufen, so dass auch in diesen Regionen eine kostengünstige Versorgung unmöglich werden wird.

Notwendig wäre schließlich eine Neuorganisation des Medikamenten- und Gerätevertriebs (z.B. die Abschaffung der PharmavertreterInnen), andere Vertriebswege als Pharmagroßhandel und Apotheken. Dabei kann es nicht um eine weitere Kommerzialisierung und Privatisierung gehen. Denkbar wäre es, die Medikamentenverteilung von den kommunalen Gesundheitszentren, die weiter unten beschrieben werden, durchführen zu lassen.

Im Pflegebereich haben wir es mit anderen Abhängigkeiten zu tun. Pflege ist ein integrativer Bestandteil jedes Ansatzes von Gesundheitspolitik und auch jeder Form von Gesundheitswesen, obwohl sie von allen Beteiligten, seien es Leistungserbringer, Kostenträger, PatientInnen oder auch die Pflegenden selbst, als ein quasi besonderer Teil gedacht wird: als etwas Zusätzliches, als eine Art Dienstleistung für die Medizin oder eine Ergänzung zu historisch - und immer noch - innerhalb von Familien organisierter Hilfeleistung bei Bedürftigkeit. Auf Grund dieser Sichtweise der Pflege als etwas nur Additivem ist ihr insbesondere in den deutschsprachigen Ländern nie eine eigenständige Funktion im Gesundheitswesen zugedacht worden, geschweige denn, dass sie als ein Bereich wahrgenommen wird, der im Mittelpunkt anderer Konzepte von Gesundheitsversorgung stehen könnte.

6 Das Institut erstellt unabhängige Gutachten zu Arzneimitteln und nicht medikamentösen Behandlungsmethoden und sieht es als seine Aufgabe, allgemeinverständliche Gesundheitsinformationen für alle zur Verfügung zu stellen. 
Solche Zuschreibungen konnten vorgenommen werden, weil es sich um einen Frauenberuf handelt, dessen Ausdifferenzierung aus dem familiären Kontext und damit eine tatsächliche Verberuflichung bis heute nicht gelungen ist. Schließlich werden auch heute noch mehr als 80 Prozent aller pflegebedürftigen Menschen in der Familie gepflegt, d.h. von Frauen, Müttern, Schwestern, Schwiegertöchtern und zunehmend von schlecht bezahlten, oft unqualifizierten Frauen aus Osteuropa. So hat die Einführung der Pflegeversicherung, von der eine enorme $\mathrm{Zu}$ nahme an Arbeitsplätzen in der ambulanten Pflege erwartet wurde, dazu geführt, dass Anspruchsberechtigte nicht Pflegeleistungen, sondern Geldleistungen beantragten, mit der die anverwandten Frauen entschädigt werden konnten. Damit konnten immerhin große Zahlen von Frauen aus den Zahlen der Arbeitslosen und Arbeitsuchenden herausgerechnet werden, wenn auch keine Arbeitsplätze mit angemessener Entlohnung für sie geschaffen wurden. An dem Beispiel wird besonders deutlich, wie wichtig es ist, Sozial- und Gesundheitspolitik von der Infrastruktur als dem allgemeineren Begriff und nicht nur von einem Grundeinkommen her zu denken: Eine Gesundheitsversorgung als Bestandteil einer steuerfinanzierten Sozialpolitik mit einem garantierten, ausreichenden Grundeinkommen birgt schnell das Risiko, Pflege als Aufgabe von weiblichen (Familien-)Angehörigen mit den bekannten Folgen zu zementieren. Eine emanzipative soziale Infrastruktur muss diese Tendenz immer wieder mitdenken und Alternativen ausloten.

Eine alternative Gesundheitspolitik, die als erstes die Gesunderhaltung, als zweites die Wiederherstellung von Gesundheit und als drittes eine menschliche Begleitung und Betreuung von unheilbar Kranken, Behinderten und Sterbenden zum Ziel hat, kann nur formuliert werden, wenn sie die genannten Faktoren kritisch einbezieht. Das heißt dann aber, Pflege anders zu definieren, als es gemeinhin getan wird. Um eine neue Definition zu finden, muss einerseits analysiert werden, wo welche Art von Pflege geleistet wird. Andererseits ist dann aufzuzeigen, wie Pflege andere Strukturen im Gesundheitswesen ermöglichen kann.

Ein immer kleiner werdender Bereich von Pflege findet in der Akutversorgung in Krankenhäusern im Anschluss an chirurgische Eingriffe statt, wobei der Schwerpunkt nicht auf der persönlichen Betreuung, sondern immer stärker auf der Kontrolle von Überwachungsgeräten liegt - ein Sektor, den es auch in einem anderen Gesundheitssystem geben wird. Dabei kann diese Art der Versorgung problemlos an technische Assistenzberufe (z.B. operationstechnische Assistenz) übertragen werden, während die psychosoziale Versorgung eher von ambulanten Pflegepersonen übernommen werden sollte, die anschließend die Nachversorgung zu Hause übernehmen werden.

Ein weiterer Pflegesektor ist die eben erwähnte ambulante Pflege, die kontinuierlich expandiert und immer mehr die frühere Krankenhausversorgung, aber auch Teile der Altenpflege übernimmt, die seit einigen Jahren verstärkt auf den ambulanten Bereich verlegt werden. Zum anderen tritt sie als komplementäres oder ersetzendes Angebot zur Pflege durch Angehörige auf. Damit wird sie zu einer wichtigen Vermittlungsinstanz zwischen medizinischer Versorgung und sozialer Betreuung, eine Funktion, die sie zu einem zentralen Bereich in einer anderen Gesundheitsversorgung prädestiniert.

Der letzte wichtige Pflegebereich ist die Betreuung chronisch kranker, behinderter oder pflegebedürftiger Menschen als Langzeitpflege in stationären Einrichtungen. Diese Versorgung ist seit langem weitgehend privatisiert und damit abhängig von der Kaufkraft der zu Versorgenden bzw. ihrer Familien äußerst unterschiedlich gestaltet. Hinzu kommt, dass dieser Bereich vorwiegend auf die Betreuung alter pflegebedürftiger Menschen zugeschnitten ist, weniger jedoch 
auf die Versorgung von Kindern, jungen Erwachsenen oder Erwachsenen, die in

Folge von Unfällen oder schwerer, nicht heilbarer Erkrankungen pflegedürftig werden und die dank einer guten medizinischen Unterstützung trotz gesundheitlicher Beeinträchtigungen eine hohe Lebenserwartung haben und damit wesentlich länger als ältere Menschen in solchen Einrichtungen versorgt werden müssen. Insbesondere in diesem Bereich gilt es, neue Strukturen zu denken, denn sowohl für die Betroffenen, für ihre Angehörigen und für diejenigen, die die Pflege leisten, sind die aktuellen Verhältnisse in der Regel unerträglich. ${ }^{7}$

Einen Sonderfall stellt die gesundheitliche Versorgung in hoch spezialisierten Privatkliniken für Privatpatienten dar, die im heutigen Gesundheitssystem eine wachsende Bedeutung erhalten. Da sie profitorientiert und zumeist hoch profitabel arbeiten, findet Pflege dort unter optimalen Bedingungen, bei guter Bezahlung und ausreichender Personalausstattung statt, weil die Pflege dort als eines der herausragenden Leistungsmerkmale mit hohen Service-/Hotelcharakter verkauft wird. Es versteht sich, dass dieses Beispiel nicht verallgemeinerungsfähig und auch nicht das Vorbild für das ist, was allen als Infrastruktur geboten werden sollte.

Insgesamt wird die Struktur des bestehenden Gesundheitssystems sowohl inhaltlich als auch finanziell faktisch durch ein Anbieterkartell beherrscht, das seine Klientel sozusagen autonom und weitgehend unkontrolliert bewirtschaftet. Die Pflege hingegen ist fast vollständig privatisiert, und zwar in Hinblick auf die Durchführung als auch auf die Bezahlung von Pflegeleistungen. Damit rückt sie zunehmend ins Visier aller Leistungsanbieter, die differenzierte Versorgungen anbieten, die sich immer mehr nach den Einkommen der Betroffenen und ihrer Angehörigen richten und nicht nach den Sätzen der Pflegeversicherung.

Als gelegentlich konkurrierender, in der Hauptsache aber ergänzender Bereich ist die Wellness-, Naturheilkunde- bis Esoterik-Industrie dazugekommen, die „Alternativen“ zu der naturwissenschaftlichen „Maschinen-Medizin“ anbietet und Ärzten wie Patienten das Leben nicht unbedingt leichter macht. Diese TeilIndustrie profitiert vor allem von den Defiziten der naturwissenschaftlichen $\mathrm{Me}$ dizin in der "Ganzheitlichkeit“ der Behandlung und natürlich von der immer wieder auftretenden Hilf- und Ausweglosigkeit in Fragen von Gesundheit, Krankheit und Sterben.

Das strukturell ungleiche Verhältnis zwischen ExpertInnen und Betroffenen macht eine Demokratisierung im Gesundheitsbereich besonders schwierig. Jedenfalls ist von Versuchen, das Verhältnis stärker zu einer „Kunden“-Beziehung umzugestalten, nichts zu erwarten. Kunden werden hier ebenso wie in anderen Bereichen von den einschlägigen Industrien dazu veranlasst, die durch Werbung und PR vor-verkauften Waren und Dienstleistungen an den Verkaufsstellen (in anderen Bereichen gern und zutreffend „outlets“ genannt) abzuholen, im Fall von Medizin-Waren und -Diensten einzufordern. Auch Patienten-Vertretungen und Selbsthilfe-Gruppen können von der Industrie und ihren Experten (durch Informationsmaterial, Vorträge, Veröffentlichungen etc.) beeinflusst, wenn nicht sogar organisiert und gesteuert werden - und das noch leichter und manipulativer als die ÄrztInnen und ApothekerInnen. Im Interesse der Heilung von Krankheiten werden gerade von Patienteninitiativen und Selbsthilfegruppen Medika-

7 Es gibt inzwischen eine Diskussion über eine neue Arbeitsteilung im Krankenhaus, die über die Delegation von ärztlichen Leistungen an Krankenschwestern geführt wird, die Teile ihrer Aufgaben an Hilfskräfte weitergeben. Der Zweck dieser Delegation ist allein die Kostenersparnis. Im Kontext dieser neuen Definitionen von Aufgabenfeldern bietet sich die Frage nach einer vernünftigen Arbeitsteilung im Krankenhaus bzw. im Gesundheitswesen an. Dabei muss im Vordergrund stehen, wer welche Leistungen am besten übernehmen kann und nicht, wer dieses am kostengünstigsten tut. 
mente und Behandlungen eingefordert, deren Sinnhaftigkeit erst noch bewiesen werden muss. Nicht zuletzt erhalten die Initiativen, die sich um solche Erkrankungen bemühen, die in der Öffentlichkeit besonderes Mitgefühl erregen oder aber den Interessen der Pharmaindustrie entgegenkommen, mehr finanzielle und materielle Unterstützung als viele andere. Insofern ist es problematisch, in den bestehenden Patientenvertretungen prinzipiell Repräsentanten allgemeiner $\mathrm{Pa}$ tienteninteressen sehen zu wollen.

Es wäre notwendig, systematisch Bedingungen dafür zu schaffen, dass eine ausreichende Aneignung von Kompetenzen auf Seiten der PatientInnen möglich wird. Das Verhältnis zwischen ÄrztInnen und PatientInnen müsste bestmöglich dem Prinzip informierter Übereinstimmung („preliminary informed consent“) folgen, was sowohl entsprechende Fähigkeiten der MedizinerInnen als auch der zu Behandelnden voraussetzt. Um Patienteninteressen zur Geltung zu bringen, genügt es nicht, die Kommunen von einer Selbstverpflichtung zu überzeugen, z.B. Gesundheitskonferenzen einzurichten. Diese Strategie ist Mitte der 80er Jahre im Rahmen der „Gesunde-Städte-Initiative“ der WHO verfolgt worden und sollte Bürger, Gewerkschaften, Verwaltungsbeamte und Arbeitgeber an einen Tisch bringen; Ärzte und Pflegekräfte sollten dabei als Berater fungieren. Wirkungsvoller ist es, ähnlich der englischen Praxis, die Mitentscheidung von BürgerInnen gesetzlich zu verankern. Mit dem Konzept der „Patient Partnership“ stehen sich Laien und Professionelle nicht mehr länger als „Nutzer/ Kunden“ und „Anbieter“ gegenüber, sondern als gleichberechtigte Parteien. So werden dort neue ambulante Einrichtungen nur noch dann staatlich gefördert, wenn die Bürger nachweislich bei Planung und Durchführung beteiligt waren.

Die sich hierzulande erst aufbauende und daran angeschlossene Schadenersatz-, Kunstfehler- und sonstige Anwalts-Teilindustrie (in den USA kann man ihre volle Wirksamkeit beobachten) ist für viele vermeidbare Untersuchungen und einiges an Bürokratie im Arzt-Patienten-Verhältnis, aber kaum für erhöhte Sorgfalt verantwortlich. Eine gemeinsame Orientierung auf das Pflegen von Gesundheit und das kluge Management von körperlichen Beeinträchtigungen statt auf die möglichst effiziente „Reparatur“ von Schäden und Krankheiten zu achten, bietet am ehesten eine Chance auf eine sinnvolle Kooperation. Dass der Arzt die „möglichst effiziente Reparatur“ beherrscht, ist allerdings vorausgesetzt, besonders bei hoch komplizierten operativen oder medikamentösen Eingriffen, aber auch schon bei dem berühmten entzündeten Blinddarm dessen, der die „Maschinen-Medizin“ kritisiert.

Es gibt einen sozialmedizinischen Konsens, dass alle Präventions-Politik (selbst wenn sie gesamtstaatlich stattfände) eine lokale und regionale Grundlage braucht. Ähnlich muss jede soziale, daher auch medizinische Versorgung der Bevölkerung lokal und regional stattfinden. Von beiden Seiten her bieten sich „lokale Gesundheitszentren" als Organisationseinheit an.

\section{Lokale Gesundheitszentren}

In der Versorgung können lokale Gesundheitszentren die Funktionen wiederbeleben, die einst der häufig mythologisierte Gemeinde- und Landarzt oder auch die Gemeindeschwester in der DDR hatte. Deren Tätigkeitsbilder als an Gesundheitsfragen orientierte Gemeinwesenarbeit ist wohl bis heute teilweise eine Motivation für die Wahl des Arzt-Berufs. Die Schwierigkeit, LandärztInnen zu gewinnen, während es in der Stadt eher ein Überangebot gibt, spricht allerdings dafür, dass diese Art von Romantik in den Köpfen und in der Realität zurückgeht. 
Es ist keine falsche Idee, dass man in Gesundheits- wie Krankheitsfragen stabile, nicht hoch spezialisierte ExpertInnen zur Verfügung haben sollte, die die Person und ihre Geschichte kennen und mit denen man sich entsprechend „ganzheitlich“ beraten kann, die aber auch über die Spezialeinrichtungen der Medizin informiert sind und bei deren Benutzung beraten und unterstützen können. Es ist ebenso sinnvoll, dass GemeindeärztInnen oder auch niedergelassene Pflegende, die neben dem Medizin-/Pflegestudium eine Public Health-Qualifikation haben müssten, sich auch für die Gesundheitsgefährdungen interessieren, die es in der Gemeinde gibt, und die PatientInnen darauf aufmerksam machen müssten. ${ }^{8}$ Die Stabilität einer solchen Beziehung zwischen PatientInnen und den sie betreuenden Fachpersonen (im Gesundheitsjargon: CasemanagerInnen oder GatekeeperInnen) wird gewiss durch die zunehmende Mobilität der PatientInnen wie des Gesundheitspersonals erschwert. Sie besteht aber andererseits für viele Leute sehr wohl, für noch mehr jedenfalls in den wichtigen Phasen von Kindheit und Alter. Im Gegensatz zur Einzelpraxis-Situation, in der vereinzelte ÄrztInnen vereinnahmt und überfordert und so zu Außenposten der Industrien und der Verwaltungen, nicht zuletzt der Krankenkassen gemacht werden, die zugleich noch unternehmerisch darauf achten müssen, dass auch für sie persönlich „die Kasse stimmt“, kann ein lokales Gesundheitszentrum einige Bedingungen für eine sachliche Arbeit herstellen helfen: Dort wären mehrere ÄrztInnen mit unterschiedlichen Spezialisierungen und mit einem gemeinsamen Maschinenpark kooperativ tätig. Darüber hinaus wäre die Zusammenarbeit zwischen ÄrztInnen, Pflegenden, TherapeutInnen, PharmazeutInnen usw. unmittelbar sichergestellt. Auch eine Koordination mit örtlichen sozialen Diensten, besonders den mobilen, wäre leicht herzustellen. Durch die Möglichkeit der leichten kollegialen Konsultation und unmittelbaren Überweisung kann das Zentrum schneller das diagnostisch Notwendige erledigen und dem sonst als „Einzelkämpfer“ tätigen betreuenden Arzt die kollegiale „Rückendeckung“ geben. Schwer, allein schon terminlich für die PatientInnen, fachlich für die ÄrztInnen zu koordinierende Überweisungen, Doppelbehandlungen und Geräteüberkapazitäten, die im bestehenden System die Regel sind, können dadurch vermieden werden. Therapieund das heißt Medikamenten-Entscheidungen können kooperativ gefällt werden. Dazu besteht eine erweiterte Möglichkeit, mobile Dienste der Therapie und häuslichen Pflege anzuschließen, sie der sozialen Situation der Patienten anzupassen und mit dem betreuenden Arzt bzw. der betreuenden Pflegeperson zu koordinieren. Wenn ein Potential der Einsparung stationärer Aufenthalte besteht, wird das der Weg sein, es zu realisieren. Dass die Dienste der Schwangerenund Mütterberatung, die amtsärztlichen Funktionen, dazu Erziehungsberatung und verwandte Dienste dort angesiedelt sein werden, versteht sich. Auch die Distribution von Medikamenten und Heil-/Hilfsmitteln über diese Zentren würde einen rationaleren Umgang mit diesen ermöglichen und die uneingeschränkte Macht der Apotheken und der Pharmaindustrie in Frage stellen.

Lokale Gesundheitszentren unter dem Gesichtspunkt der Versorgung einzurichten, ist keineswegs unerhört. Man kennt sie in verschiedenen Ländern. Auch

8 Da die Rolle der ÄrztInnen weiterhin zentral bleiben wird, stellt eine Neuorientierung der medizinischen Ausbildung einen ganz wichtigen Bestandteil einer Umstrukturierung des Gesundheitssystems dar. Die psychosoziale und sozialmedizinische Ausbildung müsste verstärkt und das naturwissenschaftlich verkürzte Verständnis vom Verhältnis Krankheit / Gesundheit / Individuum / Medizin revidiert werden. Es gälte, nicht nur individuelles Verhalten, sondern soziale Verhältnisse zum Ausbildungsinhalt zu machen. Die Verbindung von ärztlicher und sozialarbeiterischer Ausbildung müsste enger werden. Ärztliche Fortbildung darf nicht durch die Pharma-Industrie finanziert und organisiert werden. Ähnliche Überlegungen gelten auch für die Ausbildung des Pflegepersonals. Die Arbeitsteilung zwischen beiden Gruppen und anderen Berufen müsste ebenfalls neu zur Debatte stehen. 
bei uns war in der Weimarer Republik die Organisationsform der „Ambulatorien“ schon stärker entwickelt, bevor sie zum "Schutz“ der niedergelassenen Ärzte zurückgedrängt wurde, wie es nach der Vereinigung der beiden deutschen Staaten dann auch mit den in der DDR üblichen Polikliniken wiederholt wurde. Bei den Ambulatorien handelte es sich um kasseneigene Dienste, in denen Ärzte als Angestellte ambulant behandelten. Zur Aufrechterhaltung der Versorgung während des "Generalstreiks" der Ärzte vom November 1923 bis zum Januar 1924 richteten die Kassen solche Ambulatorien ein; sie stellten aber auch ein systemalternatives Versorgungsmodell zur freiberuflichen Tätigkeit von Kassenärzten dar. Die konservative Ärzteschaft sah in den Ambulatorien eine "Kampfmittel gegen die ärztliche Berufsfreiheit". 9

Wie die Entstehung von Gemeinschaftspraxen, Ärztehäusern und neuerdings auch Medizinischen Versorgungszentren (MVZ) zeigt, besteht in Teilen der Ärzteschaft selbst durchaus Interesse an solchen kooperativen Organisationsformen. Sie zu fördern und zu ermutigen, könnte schon einen Teil des Übergangs ermöglichen.

Die Strukturen dafür wurden im ambulanten Sektor inzwischen geschaffen: Leistungserbringer können heute Medizinische Versorgungszentren (MVZ) einrichten, in denen ÄrztInnen als Angestellte arbeiten können. Im ersten Quartal 2009 gab es lt. KBV ca. 1250 MVZ mit insgesamt ca. 5850 Ärzten. $52 \%$ von diesen MVZ sind in der Trägerschaft von Vertragsärzten und $38 \%$ in der Trägerschaft von Krankenhäusern. Diese MVZ bieten sich in der Tat an, wie eine Art Poliklinik bzw. lokales Gesundheitszentrum genutzt zu werden - wenn sie nicht unter den aktuellen politischen und ökonomischen Bedingungen für andere Zwecke nutzbar gemacht werden sollten. Sie bieten den Krankenhäusern die Möglichkeit, den ambulanten Gesundheitsmarkt zu betreten, was ihnen zuvor verwehrt war. Das allein wäre als eine Konkurrenz zu den bestehenden ambulanten Strukturen durchaus begrüßenswert. Zugleich aber sind mit diesen Strukturen für Konzerne wie Helios, Rhönklinikum AG, Asklepios etc. die Türen geöffnet, im ambulanten Sektor Fuß zu fassen. Damit wird der ambulante Bereich für die großen Konzerne und Aktiengesellschaften zugänglich. Die enge Verzahnung von stationärer und ambulanter Versorgung, die zwar wünschenswert ist, dient unter diesen Vorzeichen ausschließlich der Monopolisierung der Gesundheitsversorgung. Im stationären Sektor bietet dazu das Konzept des Klassenlosen Krankenbauses („Hanauer Modell“) Anregungen, das Anfang der 70er Jahre vor allem von Hans See und Martin Woythal entwickelt wurden. Zentrales Anliegen ist dabei, das privatwirtschaftliche Profitinteresse aus dem Krankenhaus zu verbannen und ein integriertes Gesundheitssystem zu schaffen. Um die Grenze zwischen ambulanter und stationärer Versorgung aufzuheben (und damit das Monopol der niedergelassenen Ärzte aufzubrechen), sieht die Planung die Einrichtung von „Polikliniken“ an jedem Krankenhaus vor, die die Nahtstelle zwischen beiden Versorgungsarten bilden. In kleineren Gemeinden müsste es Gruppenpraxen geben, in denen Allgemeinmediziner, Fachärzte, Sozialarbeiter und Psychologen arbeiten. Zentrale Merkmale des neuen Krankenhauses wären:

- Alle zahlen den gleichen Pflegesatz.

- Die Beseitigung der Chefarzthierarchie und die Einrichtung einer Krankenhauskonferenz, in die die Mitarbeiter des Krankenhauses bis hin zum Techniker, Verwalter, Krankenhausträger und auch ein Patientenanwalt nach einem bestimmten Schlüssel gewählt werden. Jeder Facharzt und jede examinierte Pflegekraft trägt die volle Eigenverantwortung.

9 Siehe Hans-Ulrich Deppe, Krankheit ist ohne Politik nicht heilbar, Frankfurt/M. 1987, S. 25. 
- Die Abschaffung des privaten Liquidationsrechts.

- Die kassenärztliche Vereinigung würde ihren Sicherstellungsauftrag und das ärztliche Behandlungsmonopol aufgeben müssen.

Sees Modell der Polikliniken kommt unseren Vorstellungen von lokalen Gesundheitszentren sehr nahe. So betrachtet wären sie nicht viel mehr als die zeitgemäße, sozialmedizinisch aufgeklärte und insofern allerdings auch notwendige Weiterentwicklung und Rationalisierung des traditionellen Modells der ärztlichen Praxis.

Pflege und der Umgang mit länger andauernden Beeinträchtigungen ist ein weiterer und immer wichtiger werdender Bereich des Gesundheitssystems, deren Organisation und Koordination zu den zentralen Aufgaben von „lokalen Gesundheitszentren “ gehört. Dafür sorgen nicht zuletzt die Veränderungen der Altersstruktur und der Familien- und Lebensverhältnisse. Pflege geht über das Medizinische hinaus, insofern sie dazu beiträgt, den jeweiligen alters- und/oder krankheitsbedingten Umständen Rechnung tragend, eine vernünftige Gestaltung des Lebens zu ermöglichen.

Zugleich lassen sich aber aus den oben genannten Aufgabenbereichen der Pflege einige konkrete Versorgungsformen entwickeln, die wichtige Teile eines alternativen Gesundheitssystems ausmachen könnten. Ein Schwerpunkt liegt dabei in der Rolle, die die Pflege bei der regionalen Versorgung durch Gesundheitszentren spielen könnte. So können Pflegepersonen, die schon seit Beginn der Vernaturwissenschaftlichung der Medizin als Übersetzerinnen zwischen dem wissenschaftlichen, exkludierenden Anspruch der Medizin und den Erwartungen der Laien-Patienten fungieren, diese Mediation zwischen medizinischem Wissen und Gesundheits- und Krankheitsempfinden der PatientInnen ausbauen. Auf Grund ihrer medizinischen Kenntnisse und ihrer sozialen Kompetenzen sollte die Pflege die erste Kontaktmöglichkeit für potenzielle PatientInnen darstellen, und zwar schon im Hinblick auf die subjektive Definition von Gesundheit und Krankheit. Denn auch im Gegensatz zu AllgemeinmedizinerInnen werden Pflegende stärker als „vertraute“ Personen wahrgenommen, die den PatientInnen und Angehörigen weniger als Fremde gegenüberstehen, sondern als Menschen aus dem eigenen Umkreis, vor denen man sich nicht fürchten muss. Sie könnten also die hierarchische Beziehung zwischen Fachleuten und Laien zu einem Teil demokratisieren. Darüber hinaus haben Pflegende, die in der häuslichen/ambulanten Versorgung tätig sind, einen Einblick in familiäre Bedingungen, durch die sie krankmachende Lebens- und Arbeitsbedingungen wesentlich früher erkennen können als dies von medizinischer Seite der Fall ist. Das heißt, sie könnten dazu beitragen, den Kompetenzerwerb der PatientInnen zu unterstützen, und gemeinsam mit den Betroffenen Maßnahmen entwickeln, die vormedizinisch wirksam würden. Für die Wahrnehmung solcher Aufgaben gibt es vor allen Dingen in peripheren und semiperipheren Ländern zahllose Beispiele, in denen Pflegende zur Bewältigung des Ärztemangels sehr erfolgreich eingesetzt werden.

In diesem Zusammenhang ist es nicht unwichtig zu bemerken, dass Pflegende in der BRD keine Leistungen, Medikamente oder Hilfsmittel verordnen dürfen was auch nicht geändert werden sollte. Das heißt, die Rolle derer, die Krankheit definieren, wäre zumindest in den ersten Stadien losgelöst von der Rolle derer, die Diagnosen stellen und Therapien verordnen. Damit lässt sich der unmittelbare Einfluss der Pharmaindustrie deutlich zurückdrängen. Darüber hinaus werden in weiten Teilen der Pflegeberufe heute bereits alternative Behandlungsmethoden schon deshalb empfohlen, weil diese als Wettbewerbsvorteil gegenüber den Ärzten eingesetzt werden können. 
Die organisatorische und inhaltliche Einbindung der Pflege in die Gesundheitszentren, in denen sie selbstverständlich als gleichberechtigte Mitglieder der therapeutischen Teams arbeiten, würde gleichzeitig gewährleisten, dass sie auf Grund enger Kommunikationsstrukturen durchaus über medizinische Entwicklungen und Möglichkeiten informiert wären. Andererseits könnten sie frühzeitig mögliche epidemiologische Veränderungen kommunizieren und gemeinsam mit den Ärztinnen und den Betroffenen entsprechende Strategien zur Gegensteuerung entwickeln.

Diese Art der Gesundheitsversorgung, die den unmittelbaren Lebensbereich einbezieht, wäre in einem anderen Gesundheitssystem auch noch viel stärker auf die gesundheitliche Beratung am Arbeitsplatz auszudehnen. Auch hier könnten an die Stelle der häufig gar nicht anwesenden BetriebsärztInnen beruflich Pflegende treten, die gemeinsam mit den Beschäftigten Quellen für Gesundheitsstörungen definieren, gesundheitliche Beeinträchtigungen der unterschiedlichen Beschäftigtengruppen identifizieren und bekannt machen. Auch die Beratung und die Propagierung präventiver Veränderungen im Betrieb ließe sich auf diese Weise schneller und situationsbezogener organisieren. Außerdem sollte man sich hier - anstelle von Arbeitsmedizin - wieder auf Ansätze von Arbeitermedizin besinnen, in der die Beschäftigten Subjekt der Untersuchung und - soweit es geht auch der Behebung der Ursachen von Arbeitsbelastungen sind.

Ein Ansatz, der an Gesunderhaltung und Prävention orientiert ist, würde es ermöglichen, unterschiedliche Lebensfelder wie Kindergärten und Schulen viel stärker einzubeziehen. Eine Sensibilisierung für Gesundheitsrisiken wie auch die Durchführung präventiver Programme könnte dort ebenfalls als Teil der allgemeinen Ausbildung, aber nicht als Teil einer totalen Kontrolle erreicht werden. Auch hier bieten sich eher Pflegende als kontinuierlichere AnsprechpartnerInnen an als ÄrztInnen, denn hier geht es vor allen Dingen um Fragen von Lebensstilen und den individuellen Umgang mit dem eigenen Körper, denn um die Behandlung und das Kennenlernen verschiedener Krankheitsbilder.

Darüber hinaus muss Pflege selbstverständlich die akute Pflege nach Krankenhausaufenthalten zu Hause durchführen, da diese in Folge der inzwischen sehr frühen Entlassung aus den Krankenhäusern hoch professionelle Anforderungen stellt, die von Angehörigen oder anderen Laien nicht erfüllt werden können. Allerdings sollte in der darauf folgenden Phase ebenso wie bei Menschen, die auf Grund ihres Alters und/oder gesundheitlicher Beeinträchtigungen bis hin zu schweren Behinderungen zu Hause versorgt werden müssen, ambulante Pflege anders organisiert werden als heute. Dabei sollten beruflich Pflegende ihre Hauptverantwortung in der Unterstützung und Organisation von Pflegenetzwerken sehen, die sich auf vielfältige Weisen zusammensetzen könnten und in denen auch zu Pflegende als Hilfen für andere Pflegebedürftige mitwirken können. Wichtig beim Aufbau solcher Netzwerke wäre es, dass individuelle Wünsche und Präferenzen der Pflegebedürftigen berücksichtigt werden, und zwar nicht nur in Hinblick auf Personen, sondern auch auf den Umfang von Pflegeleistungen, der nicht einem vorgeschriebenen Automatismus unterliegen sollte. Gleichzeitig müsste garantiert werden, dass Laienpflegende, insbesondere dann, wenn es sich um nahe Angehörige handelt, vor eigenen Gesundheitsschäden (körperlich oder psychisch) geschützt werden. Die Versorgung von Pflegebedürftigen könnte somit vielmehr zu einer gemeinsamen Aufgabe werden, in denen Hilfe über die Familie und die professionelle Unterstützung hinaus garantiert wäre und die auch die pflegerischen Aktivitäten von Männern zulassen würden. Ein wichtiges Moment einer anderen Gesundheitsversorgung muss eine andere Pflege von Sterbenden und von Menschen mit schwersten gesundheitlichen Be- 
einträchtigungen sein, d.h. von solchen Menschen, die nicht in ihrem Zuhause gepflegt werden können, die sich selbst nicht mehr an ihrer Versorgung beteiligen können und die nicht einmal mehr ihre Wünsche bezüglich der Versorgung ausdrücken können. Sicherlich wird sich die Anzahl dieser Personen reduzieren, wenn mit Hilfe von Pflegenetzwerken eine komplexe ambulante Versorgung ermöglicht wird, die zu Hause und auch vermehrt in Tages- und Kurzzeiteinrichtungen von einer Vielzahl von HelferInnen durchgeführt wird und somit die in der Familie Verantwortlichen entlastet. Zunächst einmal müssen kleine, dezentrale stationäre Einrichtungen inmitten der Kommunen eingerichtet werden, so dass auch hier die Pflegenetzwerke in die Betreuung einbezogen werden könnten und die Gettoisierung beendet wird. Empfehlenswert sind kleine Wohneinheiten für nicht mehr als zehn Pflegedürftige mit angemessenen Aufenthaltsräumen für die BetreuerInnen, beruflichen und Laien. Mittlerweile gibt es zahlreiche Modelle und Experimente, in denen alternative Versorgungsarten erprobt werden, und zwar rein privat organisierte wie auch solche, die von den Kommunen unterstützt werden. Zu garantieren ist, dass in diesen Bereich hoch professionelles Personal beschäftigt wird, was nicht heißen muss, dass hier nur Pflegende mit entsprechenden Weiterqualifikationen tätig sind, sondern vielmehr müssen es Personen sein, die physisch und psychisch darauf vorbereitet sind, diese extrem belastenden Situationen so zu bewältigen, dass weder die zu Pflegenden noch sie selbst Schaden nehmen. Feststeht, dass dieser Bereich einen wesentlich höheren Finanzbedarf haben wird als heute, da in vielen solchen Einrichtungen gegenwärtig nicht gepflegt, sondern nur versorgt wird.

Was die Arbeitsverhältnisse angeht, wäre grundsätzlich davon auszugehen, dass das Gesundheitspersonal, also ÄrztInnen, TherapeutInnen, PflegerInnen usw. von den Gesundheitszentren angestellt werden. In diesem Fall würde sich die Abrechnung von Einzelleistungen erübrigen. Natürlich würden freie Arztpraxen u.ä. weiter bestehen, aber nicht mehr eine tragende Säule des Gesundheitssystems darstellen.

Da Gesundheitszentren möglichst lokal und bedarfsnah einzurichten wären, bestimmte Geräte und Spezialbehandlungsmöglichkeiten aber einen größeren Einzugsbereich erfordern, wäre an regionale Gesundheitszentren auf höherer Ebene zu denken, die in Kooperation mit den lokalen Zentren diese betreiben. Darüber hinaus müsste es eine regionale, lokale, zentrale Instanz geben, die plant, wo welche und wie viele Zentren eingerichtet werden sollten.

\section{Finanzierung}

Sozialpolitisch spannend wird diese Organisationsform durch die Steuer-Finanzierung und durch ihre Präventions-Komponente.

Die herkömmliche paritätische Beitragsfinanzierung des öffentlichen Gesundheitssystems wird mehr und mehr ausgehöhlt. Die neueste „Reform“ sieht nun eine teilweise Finanzierung durch Steuern vor, deren Umfang stetig ansteigt. Dass die herkömmliche Form der paritätischen Beitragsfinanzierung zur Disposition steht, ist spätestens mit der schwarz-gelben Regierung offenkundig, und es ist, wie gesagt, nicht unbedingt sinnvoll, sie verteidigen zu wollen. Es wäre vielmehr notwendig, das öffentliche Gesundheitssystem völlig auf Steuerfinanzierung umzustellen. Dadurch könnte die ausschließliche Belastung von Lohneinkommen aufgehoben und die Finanzierung auf eine breitere Basis gestellt werden. Dies folgt dem von uns grundsätzlich formulierten Prinzip, die soziale Sicherung von ihrer Bindung an das Lohnarbeitsverhältnis zu lösen. Steuerfi- 
nanzierung würde es auch leichter möglich machen, die finanziellen Mittel nach regionalen und lokalen Bedarfskriterien an die dezentralen Versorgungszentren zu verteilen. Dass damit die Finanzierung des öffentlichen Gesundheitssystems stärker von den jeweiligen politischen Kräfteverhältnissen abhängt, ist sicher. Allerdings wird die paritätische Beitragsfinanzierung, die das Mittelaufkommen im Wesentlichen an den von ÄrztInnen und Pharma-Industrie gesteuerten „Bedarf“ anpasst, schon heute mehr und mehr durchlöchert. Voraussetzung einer sozial ausgeglichenen Steuerfinanzierung wäre allerdings eine grundlegende Umgestaltung des Steuersystems, das einen immer regressiveren, d.h. die Ärmeren belastenden Charakter erhalten hat. So lange die Lohnsteuern der größte Batzen im Steuertopf sind, hängt die Finanzierung auch wieder stark von Lohneinkommen ab. Eine Steuerfinanzierung des Gesundheitswesens macht daher nur Sinn, wenn die Einkommen- und Gewinnsteuern drastisch erhöht werden, die Vermögensteuer wieder eingeführt und die Erbschaftsteuer angehoben wird. Damit würden alle Mitglieder der Gesellschaft entsprechend ihrer Einkommens- und Vermögensverhältnisse an der Finanzierung der Gesundheits-Infrastruktur beteiligt, und ihre Nutzung würde allen offen stehen. Private Versicherung käme dann nur noch für besondere Zusatzleistungen, etwa im so genannten WellnessBereich oder für Präparate, für die gleichwertige oder billigere Ersatzstoffe vorhanden sind, in Frage.

Steuer-Finanzierung der Gesundheits-Sicherung heißt, dass die Krankenkassen, wenn überhaupt, dann nur in einem Teil ihrer Funktionen erhalten bleiben und in einem anderen Teil, dem des Eintreibens der Beiträge, ersatzlos auslaufen. Dasselbe gilt für die kassenärztlichen Vereinigungen. Natürlich ist weiterhin eine Verwaltung der Finanzierung nötig, und auch eine Kontrolle der Einzelleistungen, die erbracht werden, wird sich nicht ganz erübrigen. Aber zweifellos wird Gesundheitsvorsorge als Infrastruktur viele Leistungen enthalten, die sich nicht einzeln zurechnen lassen und die daher pauschal finanziert werden müssen. Die Finanzierung der lokalen Gesundheitszentren wird aus zentralen Mitteln unter Berücksichtigung regional unterschiedlicher Bedarfslagen erfolgen (wie z.B. in England praktiziert). Indem ein Teil der Finanzierung aus den Steuereinnahmen der Gemeinden erfolgt, entstehen dort Motive, an einer möglichst effektiven lokalen Gesundheitspolitik und Patientenversorgung mitzuarbeiten. Vergütungsverfahren für Einzelleistungen, soweit noch vorhanden, müssten so gestaltet werden, dass das ökonomische Interesse nicht mehr Ausmaß und Formen der Behandlung bestimmt. Wenn Finanzierung und Verwaltung völlig auf diese Zentren übergehen, werden Krankenkassen und kassenärztliche Vereinigungen überflüssig.

\section{Demokratische Selbstverwaltung}

Wenn die lokalen Gesundheitszentren mehr sein sollen als Einrichtungen einer guten und effizienten Versorgung der Bevölkerung im Krankheitsfall, wenn sie für die Erhaltung der Gesundheit zuständig sein sollen, müssen sie Aufgaben auf Dauer stellen, die heute im Rahmen von Bauaufsicht und Betriebszulassungen, zum Teil aber auch gar nicht oder von privaten und Bürger-Initiativen geleistet werden. Sie müssen imstande sein, mögliche Gefährdungen der Gesundheit durch Wirtschaft und Verkehr zu erkennen und abzustellen und Planungen so anzuleiten, dass die Bedingungen eines gesunden Lebens gesichert werden. Aus der Patientenversorgung können dazu Informationen anfallen, aber überwiegend werden sie aus anderen Bereichen der Verwaltung kommen oder aktiv gesucht 
werden müssen. Die Gesundheitszentren werden also einerseits Fachleute für Sozial-, Arbeits- und Umwelt-Medizin haben und andererseits in breitere Planungsprozesse eingebunden sein müssen. Umgekehrt werden solche übergreifenden Gremien auf die Arbeit der Gesundheitszentren Einfluss haben. Gesundheit wird damit organisatorisch zu einer Aufgabe von Gemeindepolitik überhaupt gemacht, und das in beiden Richtungen. Politik hat die Bedingungen für den Erhalt der Gesundheit herzustellen und sie hat dafür zu sorgen, dass die trotzdem notwendige Versorgung von Schäden und Krankheiten in den Rahmen der allgemeinen Gemeindepolitik gestellt wird. Dazu müssen rechtliche Bindungen der Kommunen geschaffen werden, die sie zur Kooperation mit Laien und Professionellen verpflichten.

Ganz entscheidend ist, dass die Gesundheitszentren mit demokratischen Selbstverwaltungs-Strukturen ausgestattet werden (vgl. dazu z.B. Schweden). Grundsätzlich muss das Gesundheitssystem so organisiert sein, dass die Interessen der Betroffenen gegenüber Gesundheitsexperten und Unternehmen klarer zur Geltung kommen. Allerdings bleibt Expertenwissen notwendig, und Patientenbedürfnisse können durchaus, wie schon erwähnt, von der Pharma- und Geräteindustrie manipuliert werden. In den Leitungsgremien der dezentralen Versorgungszentren müssten daher auch unabhängige Experten vertreten sein - neben der Bevölkerung und relevanten Teilen der sonstigen Gemeinde-Verwaltung und -Politik. Wie deren Zusammensetzung, Auswahl und Legitimation aussehen sollte, wäre noch zu überlegen. Eine derartige Repräsentationsstruktur ist eine wesentliche Voraussetzung dafür, dass die Gesundheitsversorgung spezifischen lokalen Bedürfnissen angepasst und über Prioritäten demokratisch und nicht nach Expertenprioritäten oder ökonomischen Interessen entschieden werden kann. Sie wäre eine wesentliche Voraussetzung dafür, dass die Abhängigkeit der Patienten von den Experten zumindest relativiert wird. Dabei müssten Erfahrungen mit Methoden der „Bedarfsermittlung von unten“ (italienische Arbeitermedizin, Gewerkschaften) berücksichtigt werden. Ein wesentliches Ziel der Bismarckschen Sozialreformen, von denen die Struktur des deutschen Sozialstaatssystems bis heute bestimmt wird, war die Zerschlagung selbstverwalteter Arbeiter-Selbsthilfeorganisationen und -krankenkassen. Dem diente auch die Einführung der paritätischen Beitragsfinanzierung, die den Unternehmen eine maßgebliche Rolle im Gesundheitssystem einräumte. Damit gälte es, Schluss zu machen." 